\title{
Sentinel Lymph Node Biopsy for Ipsilateral Breast Tumor Recurrence, Technically Feasible but Influence on Oncologic Outcomes Yet to be Completely Defined
}

\author{
James W. Jakub, MD \\ Department of Surgery, Mayo Clinic, Rochester, MN
}

In this issue of Annals of Surgical Oncology, the publication from the Sentinel Node and Recurrent Breast Cancer (SNARB) study presents recommendations in the setting of failed repeat sentinel lymph node surgery. ${ }^{1}$ The authors advocate for no additional regional therapy (adjuvant radiation or axillary lymph node dissection [ALND]) if an attempt at redo sentinel lymph node biopsy (rSLNB) fails, for an ipsilateral breast tumor recurrence (IBTR). Their primary rationale for this recommendation is the risk of regional recurrence is low in the ipsilateral axillary basin under these circumstances.

An attempt at repeat regional lymph node staging following prior ipsilateral breast cancer treatment has a higher risk of failed mapping and aberrant drainage. In patients with a positive rSLNB, the incidence of an aberrant node being the site of regional metastatic disease is fairly high, at approximately $40 \%$ (range $20-72 \%)^{2-4}$ Poodt et al. previously reported a low risk of regional recurrence in the setting of a negative SLNB, ${ }^{5}$ and herein report a low risk of regional recurrence if lymphatic mapping fails. ${ }^{1}$ Importantly, if there is a regional failure, many occur outside the ipsilateral axilla (supraclavicular, internal mammary and contralateral axilla). I agree with the conclusions that failed rSLNB should not be an impetus for additional regional therapy-ALND or regional nodal radiation. An unanswered question unfortunately still vexes us: rSLNB is technically feasible, but does it offer added value to patient outcomes?

(C) Society of Surgical Oncology 2019

First Received: 22 February 2019;

Published Online: 29 April 2019

J. W. Jakub, MD

e-mail: jakub.james@mayo.edu
Sentinel lymph node surgery has been routinely adopted into clinical practice for approximately two decades. ${ }^{6,7}$ The goal in primary breast cancer is accurately identifying the first echelon lymph nodes draining the breast. Many of the initial studies were focused on the technical logistics, i.e. what to inject, where, when, and how much. It has become clear that in a setting of primary breast cancer, peritumoral, intradermal, subareolar, and periareolar injection techniques can all be performed successfully and accurately stage the ipsilateral axilla; however, following prior surgery of the breast and axilla, the lymphatic drainage patterns may be altered. The previously rich lymphatic network may also be obliterated post-radiation or sclerosed from treatment effects of prior chemotherapy. In rSLNB, drainage of the central breast mound may still drain to the axilla, but this may not reflect the lymphatic drainage from the IBTR or new breast cancer. The lymphatics that were transected at the initial surgery may have developed alternative lymphatic egress to remote sites, and, unlike in a surgically unaltered breast, the anatomic location of the lymphatic drainage may be different based on the time lapsed from the original operation, quadrant of the breast currently involved or previously manipulated, tumor drainage via superficial or deep lymphatic networks, prior radiation, extent of previous surgical lymph node basin disruption, and prior incisions. For rSLNB, the injection location likely impacts the success of identifying a hot or blue node, and an important question unique to rSLNB remains: is the lymph node identified reflective of the lymphatic drainage pattern of the tumor itself? Mapping the breast and mapping the tumor may yield different 'SLNs' and thus, unlike in primary breast cancer, the injection location in rSLN may result in completely different lymph nodes being the target. The authors previously described their technique for rSLNB as a dual-agent approach, with the Tc-99 colloid injected deep in the 
parenchyma or scar tissue around the recurrent tumor; if lymphoscintigraphy failed, a second dose was injected. ${ }^{3}$ Would a periareolar or subareolar technique have had the same low regional recurrence in the setting of failed mapping or a negative SLNB?

As noted, aberrant drainage is more prevalent in a breast/axilla that has been previously surgically violated. In this study, it is not clear what the authors mean by "the SLNB could not be harvested"? Of those who had an unsuccessful rSLNB, 26\% had successful lymphatic mapping, 39 had aberrant drainage, and 23 had successful lymphoscintigraphy that was not aberrant. I interpret this to mean 62 patients had a lymph node seen on lymphoscintigraphy that was not surgically pursued or could not be identified intraoperatively. Identifying an SLN on lymphoscintigraphy, whether it be in an aberrant basin or not, is likely technically able to be retrieved; however, the decision to do so or not is very dependent on the provider. The manuscript does not specify the reason the lymph node visualized was not harvested. Another management issue that is a judgment decision is whether or not to proceed to an ALND in the setting of a failed rSLNB. In this study, factors associated with not completing an ALND in the setting of unsuccessful rSLNB were younger patient age, no prior SLN surgery, prior ALND, prior node-positive status with the original primary tumor, and longer interval from the primary tumor to the IBTR, many of which are collinear and related to the original management of the axilla. One potentially important factor that the authors do not report is the operating surgeon. The decision to proceed with an ALND is likely driven by the surgeon's judgment and philosophy in this setting. Did the SNARB study define a best practice of how to manage the axilla in the setting of failed rSLNB? Did the SNARB study dictate that all aberrant SLNs visualized on lymphoscintigraphy be harvested? Were these decisions left to surgeon discretion over the study period?

In terms of management of the axilla at the time of failed rSLNB, the major difference was the status of the axilla at the original operation. Of the patients who proceeded to ALND if rSLNB failed, $87 \%$ had no prior axillary staging or only an SLNB at the original operation for their primary tumor. Of the group that did not proceed to ALND if rSLNB failed, $85 \%$ had a prior ALND. Thus, almost everyone in this study had an ALND, either at the primary breast operation or at the IBTR. Of the 515 patients in whom rSLNB was attempted, 239 failed; of these, we are left with only 26 patients who did not have an ALND with their primary operation or when they failed rSLNB for their IBTR. It is also worth noting that $95 \%$ of the population had prior BCT. As a result, the conclusions from this study, i.e. not to perform additional regional therapy (ALND or radiation) and the resultant low same- basin regional recurrences, cannot be applied to patients who had a previous mastectomy or to patients who underwent only an SLNB or no axillary staging at their original diagnosis.

The authors describe their repeat operations being performed for IBTR. ${ }^{1}$ Sixty percent of the patients in this study were $>10$ years from their primary breast malignancy; thus, I suspect a number of these events were new primaries. Differentiating these two can be inexact, but is more than simply an academic exercise as the prognostic significance of a new primary versus recurrence can be quite different. It is worth noting that $63 \%$ of patients did not undergo SLNB at their original operation; $67 \%$ underwent an ALND but only $21 \%$ were node-positive. This long disease-free interval and lack of SLNB would imply many primary breast cancer operations were performed at a time preceding SLNB being part of routine practice.

Unfortunately, we cannot look at regional recurrence rates in those not undergoing an ALND and draw a conclusion analogous to ACOSG Z11 based on these findings as this was not a randomized trial and, as discussed above, the decision to proceed with ALND was highly influenced by prior management of the axilla, with almost $90 \%$ of patients who did not undergo lymphadenectomy having a prior ALND at their original operation. As a result, comparing the regional recurrence rates in those with failed rSLNB of $0 \%$ in the ALND group and only $3 \%$ in the group observed, is an unfair comparison because they are very different populations with very different regional management at the time of their original cancer diagnosis. We really do not know what the regional recurrence would be in those who had no prior ALND if rSLNB was unsuccessful and no further axillary management was performed as only 26 patients met these criteria. The finding of a macrometastasis in the rSLNB clearly influenced clinical decision making as $50 \%$ of those pathologically staged as node-positive received adjuvant chemotherapy and $50 \%$ received adjuvant radiation. The percentages for those staged node-negative at rSLNB were $12 \%$ and $15 \%$, respectively. Based on the CAOLR randomized controlled trial of adjuvant chemotherapy or not for recurrent breast cancer, it could be strongly argued the decision to offer adjuvant chemotherapy should be primarily based on the tumor subtype. ${ }^{8}$

Tumor cells disseminate via a lymphatic and/or hematogenous route. Repeat SLNB may fail secondary to obliterated lymphatics from prior radiation. Failed lymphatic mapping may imply inadequate lymphatic drainage of the breast, which not only prevents small injected particles from travelling via the lymphatics but also tumor cells. Thus, the inability for lymphatic drainage, which we recognize as failed rSLNB, may be a marker associated 
with a safe haven of regional lymphatic spread, and thus a lymph node dissection in this setting would have a low pathologic yield and unlikely impact recurrence rates or direct adjuvant therapy.

The SNARB consortium from The Netherlands has further contributed to the growing body of literature regarding rSLNB. Metastatic spread beyond the primary to the regional nodal basin demonstrates a more aggressive tumor behavior. In turn, this information could identify aberrant positive lymph nodes, alter adjuvant treatment, and potentially decrease regional recurrence rates. Given the relatively low risks of repeat sentinel node biopsy, the risk/benefit ratio often favors performing rSLNB. Although I agree with their conclusion that failed rSLNB in the setting of a new breast cancer event, ipsilateral to a prior lumpectomy, does not warrant an ipsilateral ALND, unfortunately the question still lingers in my mind regarding how much value the rSLNB adds to patient outcomes. Future studies will need to define if identifying a positive or negative lymph node not only impacts the multidisciplinary team's decision to give or withhold more therapy but does the procedure and its downstream treatment implications improve patient's oncologic outcomes? Does anatomic staging in recurrent breast cancer still matter or is biology alone king?

\section{REFERENCES}

1. Poodt IGM, Walstra CJEF, Vugts G, et al. Low risk of development of a regional recurrence after an unsuccessful repeat sentinel lymph node biopsy in patients with ipsilateral breast tumor recurrence. Ann Surg Oncol. 2019. https://doi.org/10.1245/s1043 4-019-07272-4.

2. Ugras S, Matsen C, Eaton A, Stempel M, Morrow M, Cody HS. Reoperative sentinel lymph node biopsy is feasible for locally recurrent breast cancer, but is it worthwhile? Ann Surg Oncol. 2016;23(3):744-8.

3. Maaskant-Braat AJ, Roumen RM, Voogd AC, Pijpers R, Luiten EJ, Rutgers EJ, et al. Sentinel node and recurrent breast cancer (SNARB): results of a Nationwide Registration Study. Ann Surg Oncol. 2013;20(2):620-6.

4. Ahmed M, Baker R, Rubio IT. Meta-analysis of aberrant lymphatic drainage in recurrent breast cancer. $\mathrm{Br} \mathrm{J}$ Surg. 2016;103(12):1579-88.

5. Poodt IGM, Vugts G, Maaskant-Braat AJG, Schipper RJ, Voogd AC, Nieuwenhuijzen GAP. Sentinel Node and Recurrent Breast Cancer (SNARB) Study Group. Risk of regional recurrence after negative repeat sentinel lymph node biopsy in patients with ipsilateral breast tumor recurrence. Ann Surg Oncol. 2018;25(5):1312-21.

6. Krag DN, Weaver DL, Alex JC, Fairbank JT. Surgical resection and radiolocalization of the sentinel lymph node in breast cancer using a gamma probe. Surg Oncol. 1993;2(6):335-9; discussion 340.

7. Giuliano AE, Kirgan DM, Guenther JM, Morton DL. Lymphatic mapping and sentinel lymphadenectomy for breast cancer. Ann Surg. 1994;220(3):391-8; discussion 398-401.

8. Wapnir IL, Price KN, Anderson SJ, et al. Efficacy of chemotherapy for ER-negative and ER-positive isolated locoregional recurrence of breast cancer: final analysis of the CALOR trial. J Clin Oncol. 2018;36(11):1073-9.

Publisher's Note Springer Nature remains neutral with regard to jurisdictional claims in published maps and institutional affiliations. 